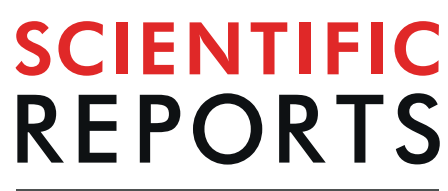

natureresearch

Received: 11 January 2019

Accepted: 21 June 2019

Published online: 29 July 2019

\section{Automated detection and classification of early AMD biomarkers using deep learning}

\author{
Sajib Saha ${ }^{1,3}$, Marco Nassisi $\oplus^{1}$, Mo Wang ${ }^{1}$, Sophiana Lindenberg ${ }^{1}$, Yogi kanagasingam ${ }^{3}$, \\ Srinivas Sadda ${ }^{1,2} \&$ Zhihong Jewel Hu $\mathbb{D}^{1}$
}

Age-related macular degeneration (AMD) affects millions of people and is a leading cause of blindness throughout the world. Ideally, affected individuals would be identified at an early stage before late sequelae such as outer retinal atrophy or exudative neovascular membranes develop, which could produce irreversible visual loss. Early identification could allow patients to be staged and appropriate monitoring intervals to be established. Accurate staging of earlier AMD stages could also facilitate the development of new preventative therapeutics. However, accurate and precise staging of AMD, particularly using newer optical coherence tomography (OCT)-based biomarkers may be time-intensive and requires expert training which may not feasible in many circumstances, particularly in screening settings. In this work we develop deep learning method for automated detection and classification of early AMD OCT biomarker. Deep convolution neural networks (CNN) were explicitly trained for performing automated detection and classification of hyperreflective foci, hyporeflective foci within the drusen, and subretinal drusenoid deposits from OCT B-scans. Numerous experiments were conducted to evaluate the performance of several state-of-the-art CNNs and different transfer learning protocols on an image dataset containing approximately 20000 OCT B-scans from 153 patients. An overall accuracy of $87 \%$ for identifying the presence of early AMD biomarkers was achieved.

Age-related macular degeneration (AMD) is the leading cause of blindness among elderly individuals in the developed world. AMD affects 1 in 7 over the age of 50, with the incidence increasing with age ${ }^{1}$. It is estimated that about 8 million people in the United States who are 55 and above years old have monocular or binocular intermediate $\mathrm{AMD}$ or monocular advanced $\mathrm{AMD}^{2}$. Advanced $\mathrm{AMD}$ is defined by the presence of central atrophy or macular neovascularization and is commonly associated with visual loss. The chances of progression to advanced $\mathrm{AMD}$ in 5 years period is $27 \%$ for patients with intermediate $\mathrm{AMD}^{3}$. For patients with already advanced AMD in the fellow eye, this chance can be as high as $43 \%^{3}$. Though treatments are now exist for patients with neovascular AMD (choroidal neovascularization, CNV), these patients likely to develop atrophy over time and appear to lose vision eventually. No proven treatment is currently available in the setting of non-neovascular disease to prevent the progression of atrophy, termed geographic atrophy (GA). Some agents under study may slow the progression of $\mathrm{GA}^{4}$, however, it is desirable to intervene in AMD patients at an earlier stage, prior to development of irreversible atrophic changes or destructive exudation from CNV. In conducting such early intervention studies, it is critical to identify those patients with high risk for progression to advanced AMD.

Historically, color fundus photograph has been the gold standard for determining early AMD. Relying on color fundus photographs, various studies ${ }^{3,5,6}$ have identified risk factors for progression that include the manifestation of large drusen, an increased total drusen area, hyperpigmentation, and depigmentation. Based on these risk factors, the Age-Related Eye Disease Study (AREDS) defined a nine-step detailed scale ${ }^{7}$, as well as a simplified scale ${ }^{8}$ for assessing the risk of progression of AMD. The simple scale used only two factors namely large drusen and pigmentary changes to assess the eye and was designed for easy clinical application. Optical coherence tomography (OCT) has largely supplanted color fundus photography in clinical practice in recent days, because OCT provides three-dimensional cross-sectional anatomic information of retinal abnormalities, which color fundus photography cannot provide. Several novel OCT-based features have been identified by a number of studies to signal risk of AMD progression ${ }^{9}$. Higher central drusen volume ${ }^{10}$, intraretinal hyperreflective foci ${ }^{11}$,

${ }^{1}$ Doheny Eye Institute, Los Angeles, CA, 90033, USA. 'Dept. of Ophthalmology, David Geffen School of Medicine, The University of California, Los Angeles, CA, 90033, USA. ${ }^{3}$ Australian e-Health Research Centre, CSIRO, Perth, Australia. Correspondence and requests for materials should be addressed to Z.J.H. (email: jhu@doheny.org) 
heterogeneous internal reflectivity within drusenoid lesions (IRDL) ${ }^{12}$, and reticular pseudodrusen or subretinal drusenoid deposits (SDD) $)^{13-15}$, are some of the promising ones that appear to signal risk for progression to advanced $\mathrm{AMD}^{9}$. OCT provides excellent opportunities to better understand $\mathrm{AMD}$ and its associated biomarkers, however, it generates massive image data volume (up to hundreds of B-scans per examination), which makes manual analysis of OCT extensively time-taking and impractical in many circumstances.

A number of approaches have already been proposed using retinal OCT images for automated and semiautomated analysis of AMD biomarkers, namely drusen ${ }^{16-22}, \mathrm{GA}^{23-26}$, pigment epithelial detachment (PED) ${ }^{27-30}$, and intra-/sub-retinal fluid ${ }^{31-33}$. Algorithms for drusen detection and segmentation ${ }^{23-26}$, primarily depend on on the difference between the actual retinal pigment epithelial (RPE) surface and a calculated ideal RPE or Brunch's membrane for automated recognition of drusen. In contrast to other methods, de Sisternes et al. ${ }^{19}$ utilized 11 drusen specific features for determining the likelihood of progression from early and intermediate AMD to exudative AMD. Information about drusen texture, its geometry, reflectivity, number, area as well as volume were used for computing the likelihood. GA detection algorithms $\mathrm{s}^{27-30}$ on OCT mainly used a partial summed voxel projection (SVP) of the choroid relying on the increase in reflectance intensity beneath Bruch's membrane in the GA. Chen et al. ${ }^{23}$ proposed a classic method in this category. The method first segmented the RPE. A partial SVP underneath the RPE was subsequently generated and the en face image was computed using the average axial intensity within the slab. Finally, GA was identified GA with the help of an active contour model and using the en face projection. Chiu et al. ${ }^{34}$ used abnormal thinning and thickening of the RPE-drusen complex (RPEDC), defined by the inner aspect of the RPE plus drusen material and the outer aspect of Bruch's membrane, to identify GA and drusen, respectively. In order to quantify PED volume in OCT, Ahlers et al. ${ }^{27}$ and Penha et al. ${ }^{28}$ relied on a similar principle as for drusen detection based on comparing the actual RPE position with the ideal or normal RPE position. To quantify PED on OCT, graph-based surface segmentation was used by Sun et al. ${ }^{29}$ and Shi et al..$^{30}$. Algorithms for intra- and subretinal fluid detection in OCT relied on a number of image analysis techniques such as gray level ${ }^{31}$, gradient-based segmentation ${ }^{32}$, active contours ${ }^{33}$, and convolutional neural networks ${ }^{35}$. Schmidt-Erfurth et al. ${ }^{36}$ proposed a method for predicting individual disease progression in AMD relying onmachine learning and other advanced image processing techniques. Imaging data that include segmented outer neurosensory layers and RPE, drusen and hyperreflective foci, together with demographic and genetic input features were used for the prediction. The method predicted the risk of conversion to advanced AMD, with area under curve (AUC) of 0.68 and 0.80 , respectively for CNV and GA. An overview and summary regarding various methods for automated analysis of AMD biomarkers on optical coherence tomography has recently been published by Wintergerst $2017^{35}$.

In our study, we report on the performance of an automated method for detection and classification of multiple early AMD biomarkers: namely, reticular pseudodrusen, intraretinal hyperreflective and hypoflective foci (Fig. 1). Worth mentioning, the proposed study has been inspired by the results of our group ${ }^{9}$ that found a great association of reticular pseudodrusen, intraretinal hyperreflective and hypoflective foci, and drusen volume with overall AMD progression. Drusen volume was the least predictive among these four biomarkers. In addition to that todays machines are already capable to perform drusen volume measurements. That is why, this paper mainly focuses on developing artificial intelligent methods for the assessment of SSD, HRF and hRF.

In clinical practice, this tool could be employed as a screening method to rapidly identify B-scans which require further attention and critical analysis by the practitioner, thus increasing the accuracy and efficiency of diagnosis.

\section{Methods}

Dataset. Spectral domain (SD)-OCT images of 153 patients who were diagnosed with early or intermediate AMD in at least one eye at the Doheny Eye Centers between 2010 and 2014, were collected and analyzed for this study. All eyes were captured using a Cirrus HD-OCT camera (Carl Zeiss Meditec, Dublin, CA) with 1024 (depth) $512 \times 128$ cube $(2 \times 6 \times 6 \mathrm{~mm})$ centered on the fovea. All images were de-identified according to Health and Insurance Portability and Accountability Act Safe Harbor prior to analysis. Ethics review and institutional review board approval from the University of California - Los Angeles were obtained. The research was performed in accordance with relevant guidelines/regulations, and informed consent was obtained from all participants. A total of 19584 OCT B-scans were available for this study and about $90 \%$ of these B-scans did not contain features of disease (i.e. were normal). In order to balance the number of disease and normal B-scans, only a portion of the normal images were used for our experiment, and concurrently data augmentation was performed for the disease cohort.

All B-scans were graded by certified expert Doheny Image Reading Center (DIRC) OCT graders. B-scans were classified as having a disease feature present only if the grader was $>90 \%$ confident that the feature was present. A total of 1050 OCT B-scans were graded as having definite subretinal drusenoid deposit, 326 B-scans had definite intraretinal hyper-reflective foci, and $206 \mathrm{~B}$-scans had definite hyporeflective drusen. In addition, subretinal drusenoid deposits, intraretinal hyperreflective foci, and hyporeflective drusen were graded to be questionably present (i.e. grading confidence of 50-90\%) in 308, 85, and 45 B-scans, respectively. As these questionable B-scans had some level of ambiguity, they were excluded from the experiment. In order to avoid any bias in training the deep $\mathrm{CNN}$, we decided to use about same number of images both for the disease and no-disease category. We performed data augmentation specifically by rotation (in the range of -5 to 5 degrees), shearing (in the range 0.2), scaling (in the range 0.2) and flipping, to increase the number of diseased images by 10 15 times. Table 1 summarizes the number of B-scans used for this experiment. $90 \%$ of these B-scans were used for training and $10 \%$ were used for testing. Training and test images were selected randomly. Further to that, training set and test set were divided prior to doing any data augmentation, to avoid data impurity. 

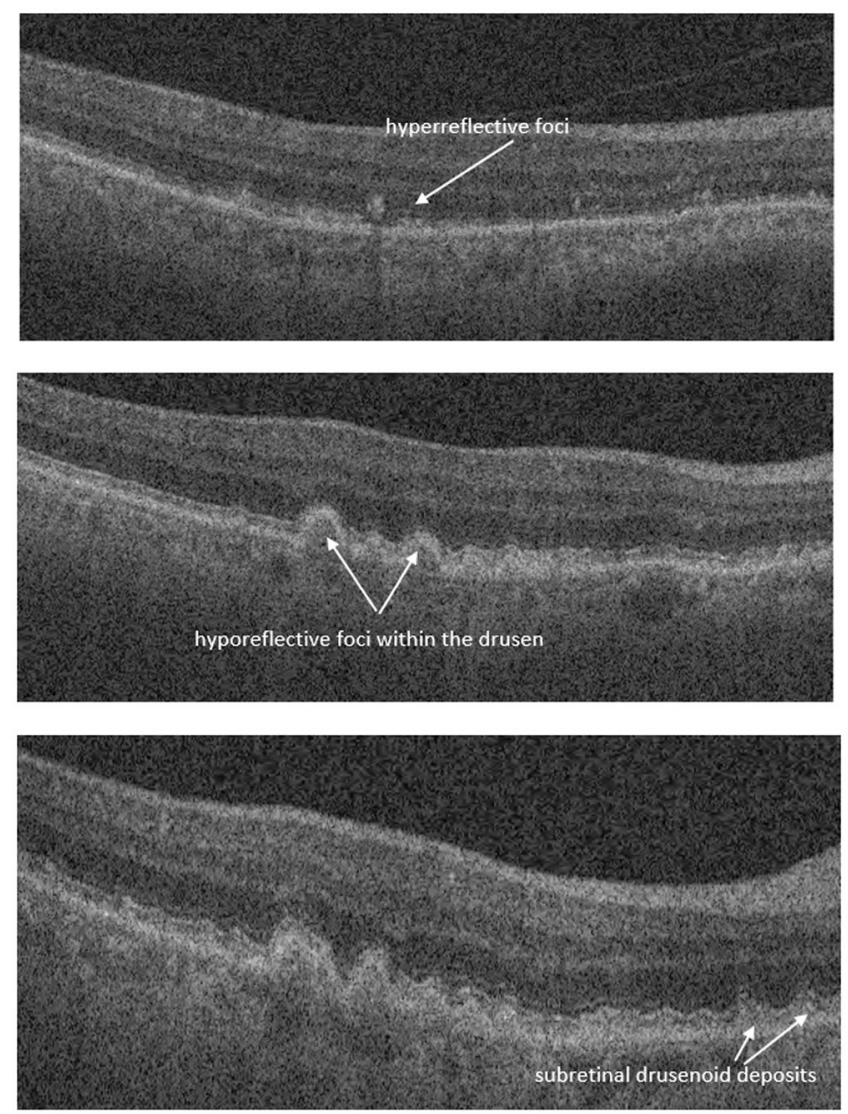

Figure 1. Example of hyperreflective foci, hyporeflective foci within drusen and subretinal drusenoid deposit.

\begin{tabular}{|l|l|l|l|l|}
\hline \multirow{2}{*}{$\begin{array}{l}\text { Early AMD } \\
\text { pathologies }\end{array}$} & \multicolumn{3}{|l|}{$\begin{array}{l}\text { No of B-scans initially } \\
\text { available }\end{array}$} & $\begin{array}{l}\text { No of B-scans used for the } \\
\text { experiment }\end{array}$ \\
\cline { 2 - 5 } & Disease & No-disease & Disease $^{\dagger}$ & No-disease $^{\Gamma}$ \\
\hline SDD & 1050 & 18222 & 10500 & 10800 \\
\hline HRF & 326 & 19173 & 4890 & 5300 \\
\hline hRF & 206 & 19933 & 3090 & 3100 \\
\hline
\end{tabular}

Table 1. Summary of the number of B-scans used for the experiment. ${ }^{\dagger}$ Following augmentation. ${ }^{\Gamma}$ Selected randomly.

Grading protocol for OCT B-scans. Each of the B-scans of the $512 \times 128$ macular cube was individually assessed to determine the presence of intraretinal hyperreflective foci (IHRF), hyporeflective foci (hRF) within druseniod lesion (DL) and subretinal drusenoid deposit (SDD) ${ }^{9}$. Drusenoid lesions typically appear homogeneous internally with a ground-glass medium reflectivity 9 . Graders explicitly looked for the occurrence of hyporeflective foci within the drusen (Fig. 1). Knowing the requirement of the presence of enough number of pixels inside a drusen to reliably determine hRF, drusenoid lesions with a height of at least $40 \mu \mathrm{m}$ was only taken into account while assessing the internal reflectivity ${ }^{37}$. IHRFs were defined as discrete, well-circumscribed hyperreflective lesions within the neurosensory retina, and a reflectivity at least as bright as the RPE band (Fig. 1) ${ }^{38}$. A minimum size of 3 pixels was set for IHRFs, to differentiate from noise and retinal capillaries. SDDs were defined as medium-reflective hyper-reflective mounds or cones, either at the level of the ellipsoid zone or between the ellipsoid zone and the RPE surface (Fig. 1) ${ }^{9}$. A lesion was considered present if the grader had greater than $90 \%$ confidence that it was present in at least one B-scan, which is the conventional practice of the reading-center?

Identifying early AMD biomarkers using deep learning. We used deep learning ${ }^{39}$ for automated identification of these OCT-based AMD biomarkers. Deep learning, also known as deep structured learning or deep machine learning, is the process of training a neural network to perform a given task ${ }^{40}$. In comparison to traditional machine learning approaches that still depend on hand-crafted features to extract valuable information from data, deep learning employs machine to learn the features by itself ${ }^{41}$. Thus, deep learning approach is more objective and robust. In addition to that, traditional machine learning approaches require manual outlining of pathology/features, which is expensive and time consuming to produce; ${ }^{42}$ whereas, deep learning requires only 


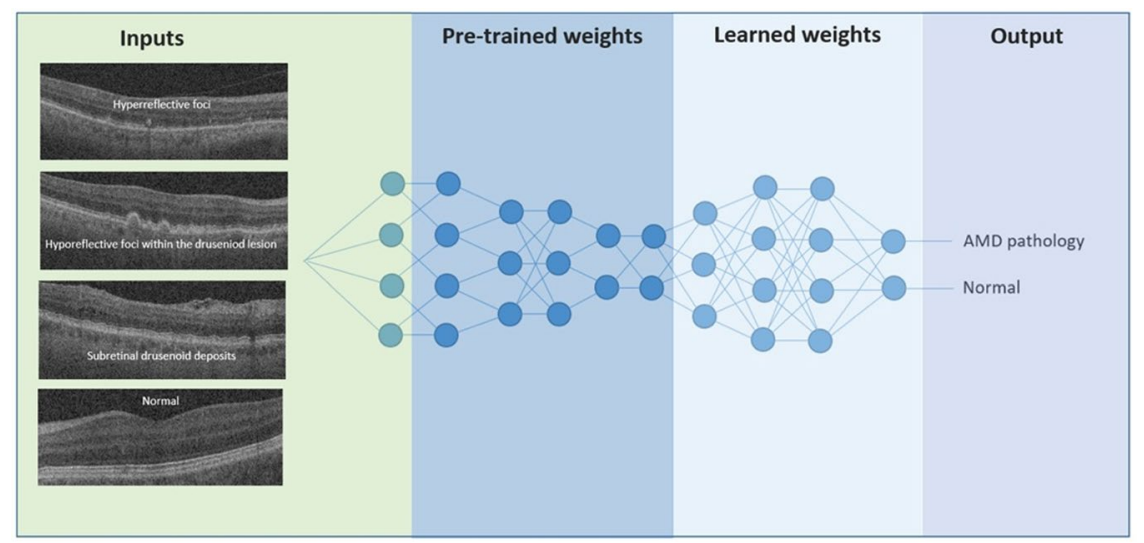

Figure 2. Deep learning for identifying the presence of early AMD biomarkers. Neuron connections shown here are for illustration only. Inspired by the schematic representation of Kermany et al. ${ }^{51}$.

the label of the data, which can be produced quickly. Importantly enough, in recent years, deep learning techniques are found to beat traditional machine learning approaches with significant margins and have become state-of-the-art in image classification, segmentation, and object detection in medical and ophthalmic images ${ }^{42}$. A problem of deep learning though, was the requirement of huge labelled data; however, through 'transfer learning $^{34}$, now it is possible to overcome this requirement. Hence, deep learning coined with transfer learning is an ideal fit in the context.

Deep convolution neural networks (CNNs) that were specially designed to process images were trained from the intensities of the OCT B-scans. During the training process we initialized the parameters of the CNN using transfer learning, as shown in Fig. 2. More specifically, we used pre-trained models that were already trained using a very large image dataset named ImageNet ${ }^{44}$ to initialize the network parameters, which were then fine-tuned using the provided image dataset. Transfer learning enables fast network training with less epochs, thus further avoids over fitting and ensures robust performance. It is a promising alternative to full training and is already applied in many areas of biomedical imaging including retinal imaging ${ }^{41,43}$.

While fine tuning the $\mathrm{CNN}$, we considered 11 different setups - fine-tuning the last $0 \%, 10 \%, 20 \%, 30 \%, 40 \%$, $50 \%, 60 \%, 70 \%, 80 \%, 90 \%$ and $100 \%$ layers. For a CNN with $L$ layers, if $\alpha_{l}$ denote the learning rate of the $l$-th layer in the network, $0 \%$ fine-tuning or fine-tuning only the last layer of the network was defined as setting $\alpha_{l}=0$ for $l \neq L$. Likewise for $P \%$ fine-tuning we train up-until $P+1$ layers.

Worth mentioning, typically, the initial layers of CNN learn low-level image features. In general, these low-level features do not vary significantly from application to application. The top layers of CNN learn high-level features, which are specific to the application at hand. Therefore, fine-tuning only the top few layers is usually sufficient while training $\mathrm{CNN}^{43}$. However, when source and target applications differ substantially, fine-tuning only the last few layers may not be sufficient. Therefore, an efficient fine-tuning strategy is to start from the last layer and then incrementally add more layers in the update process until the desired performance is reached.

For each of the pathology types we trained three different nets namely Inception-v $3^{45}$, ResNet $50^{46}$, and InceptionresNet $50^{47}$. For each net, we conducted the experiment on 11 different setups (e.g. fine-tuning strategy) as explained in 'Identifying early AMD biomarkers using deep learning. We compared the performance of different setups for all three CNNs. Experiments were conducted likewise for each of the three AMD biomarkers.

Automated segmentation of retinal layer using ReLayNet. Prior to feeding the image into CNN for pathology detection and classification, we performed a pre-segmentation of the retinal layers using ReLayNet ${ }^{48}$, as our early AMD biomarkers tend to be localized to specific retinal layers. SDD usually appear above the inner RPE surface, hyporeflective drusen are usually located above the Bruch's membrane/inner choroid surface, and hyperreflective foci may appear in several different outer retinal layers. ReLayNet produced an 8-layer segmentation mask, which were then used to compute a binary mask that only contains the retinal region spanning from the outer nuclear layer (ONL) to Bruch's membrane/inner choroid. It is worth noting that ReLayNet itself is a deep learning framework which is specially designed to perform segmentation of retinal layers in OCT B-scans. The framework is validated on a publicly available benchmark dataset with comparisons against five state-of-the-art segmentation methods including two deep learning based approaches to substantiate its effectiveness. The computed binary mask is finally imposed on the input image to define the region of interest.

The pixel level segmentation purely based on ReLayNet contained some outliers that includes small holes within the region of interest, and scattered group of pixels/small regions. We performed morphological operations including region filling and length based object removal to avoid those outliers. Figure 3 shows an example OCT B-scan, and corresponding region of interest mask generated purely based on ReLayNet and ReLayNet with other pre-processing.

Performance metrics. The performance metrics which we used included accuracy, sensitivity, specificity and area under the curve (AUC). Accuracy was defined as the ratio of the number of correct identifications made over the total number of images available on the validation set. Sensitivity was defined as the proportion of 

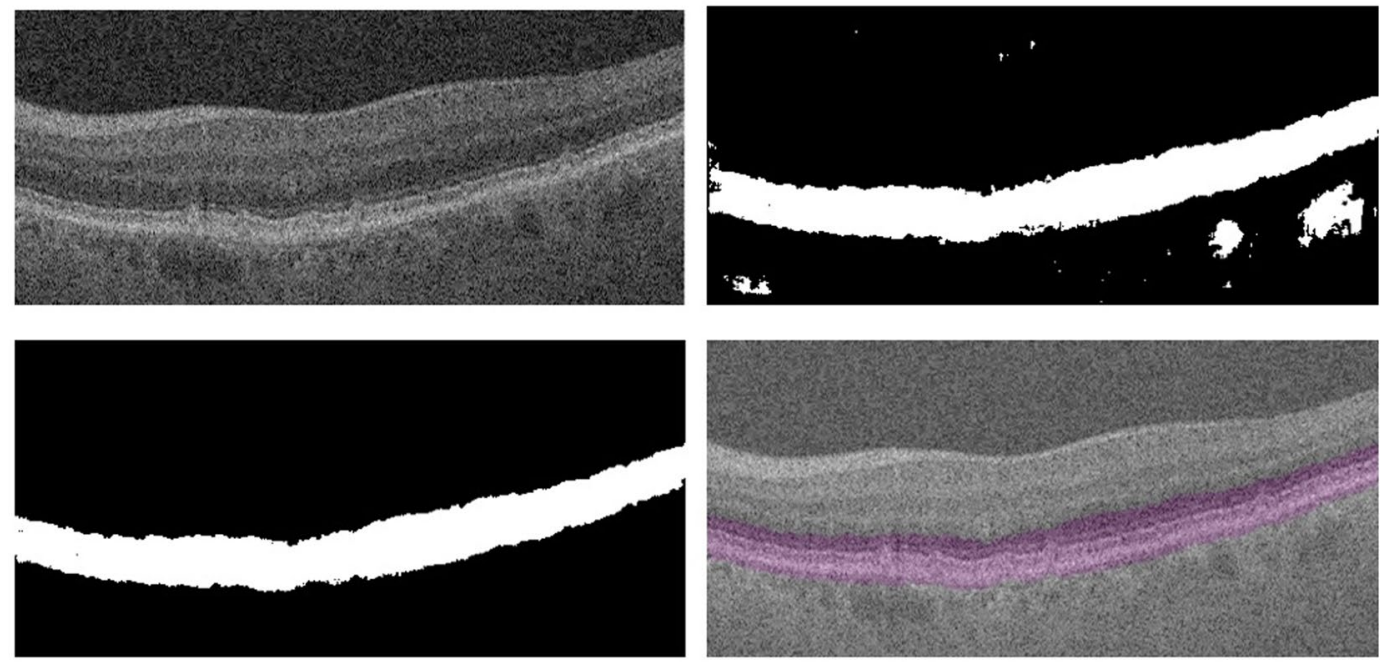

Figure 3. Top-left: an example OCT B scan, top-right: region of interest mask generated based on ReLayNet, bottom-left: region of interest mask generated using ReLayNet and other image pre-processing technique, bottom-right: mask (shown in purple) superimposed on the image.

actual positives that are correctly identified, whereas specificity was defined as the proportion of actual negatives that are correctly identified. To compare different setups and nets, and receiver operating characteristic (ROC) curves were mainly used. ROC curves plot the detection probability (i.e. sensitivity) versus false alarm rate (i.e. 1-specificity).

Use of human participants. Ethics review and institutional review board approval from the University of California - Los Angeles were obtained. The research was performed in accordance with relevant guidelines/ regulations, and informed consent was obtained from all participants.

\section{Results}

Figure 4 shows the fitted curve representing the validation accuracy over epochs by the three different nets for identifying the presence early AMD pathologies. The validation accuracy (against epochs) of the best setups are only shown.

From Fig. 4 it can be inferred that accuracy improvement gets saturated by 10 50 epochs for SDD, about 20 40 epochs for IHRF, and about 20 40 epochs for hRF. The performance between different nets are not to an extent that would be relevant in practice. However, from the receiver operating characteristic (ROC) curves as shown in Fig. 5, InceptionResNet is better suited for detecting the presence of SDD and IHRF; and Inception is better suited for identifying the presence of hRF. Table 2 summarizes the sensitivity, specificity, AUC and accuracy obtained by different models.

In aggregate, experiments on all the three CNNs show promising results on identifying the presence of early AMD pathologies. Accuracy ranged from $86 \sim 89 \%$. SDD can be identified with an accuracy of $80 \% \sim 86 \%$. Accuracy for identifying the presence of IHRF and hRF were $89 \%$ and $88 \%$, respectively. SDD can be best detected by InceptionResNet, having sensitivity, specificity and accuracy of $79 \%, 92 \%$ and $86 \%$, respectively. HRF was also best detected by InceptionResNet with sensitivity and specificity of 78\%, and 100\%, respectively. hRF was best detected by Inception with sensitivity and specificity of $79 \%$ and $95 \%$, respectively.

\section{Discussion}

We propose an automated system for identifying the presence of early AMD biomarkers from OCT B-scans. By employing transfer learning algorithm, the proposed system showed good performance for this application without the need for a highly specialized deep learning machine or a database of millions of images. The system provides numerous benefits, including consistency in prediction (because a machine will make same prediction for same image each time) and instantaneous reporting of results. In addition to that since the algorithm can have multiple operating points, its sensitivity and specificity can be adjusted to meet specific clinical requirements, for example high sensitivity for a screening application.

One fundamental limitation of deep learning were the requirement of huge number of training images. However, with the development of transfer learning paradigm, this is not a limitation any more. Relying on transfer learning state-of-the-art classification performance is achieved using only couple of hundreds to thousands of images ${ }^{49,50}$. A very relevant example is the recent study made by Christopher et al. ${ }^{50}$. Christopher et al. have used a fundus dataset of 14,822 images and relying on transfer learning, they have achieved state-of-the-art accuracy of $91 \%$ in distinguishing glaucomatous optic neuropathy eyes from healthy eyes. Our study that involved about 20,0000 OCT scans and used transfer learning is fully sufficient and the results are representative.

Although we are able to train a highly accurate deep learning model here, with a relatively small training dataset, unsurprising, it's performance would be inferior to that of a model which is trained using 'full training', or in other words from a random initialization on an extremely large dataset of OCT images. All the network weights 


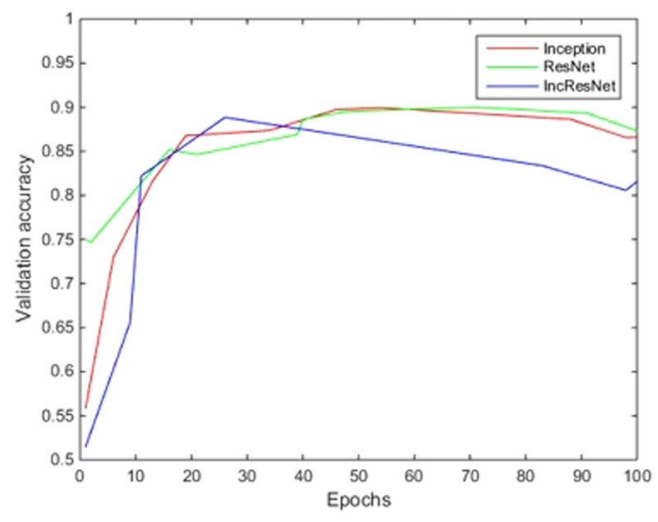

(a)

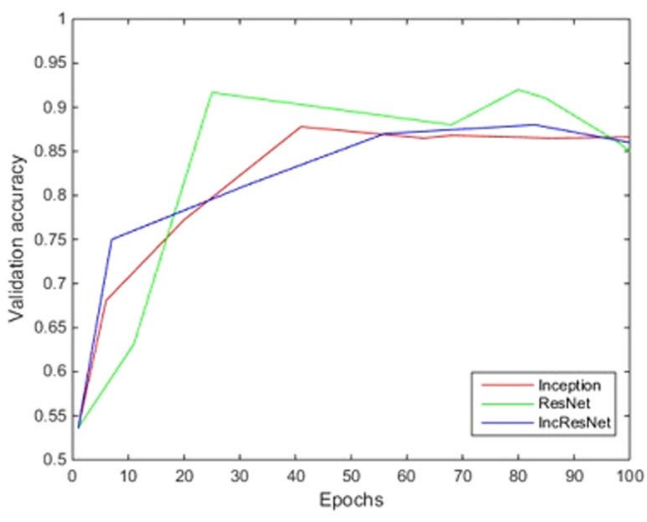

(b)

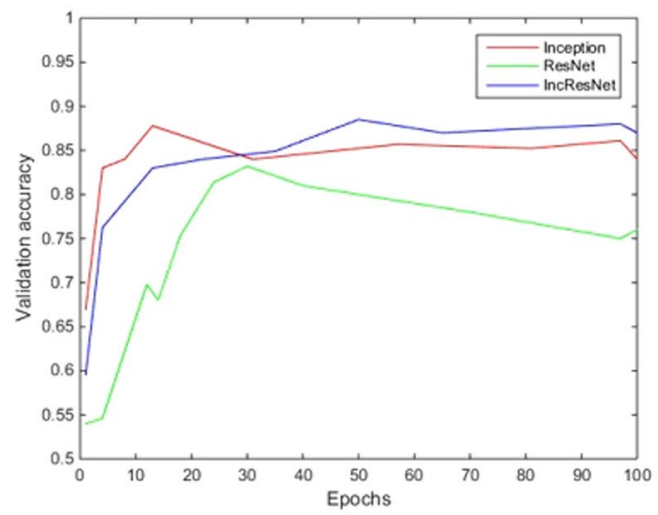

(c)

Figure 4. Fitted curve representing the validation accuracy over epochs by the three different nets for identifying the presence of (a), IHRF (b) hRF, and (c) SDD.

of the model are directly optimized when full training is performed. However, OCT images in such a volume to train a blank CNN are difficult to ascertain.

Similar to other transfer learning based models, the performance of our system depends highly on the weights of the pre-trained model. Therefore, the performance of the system would likely be enhances when more advanced pre-trained models that are trained with even larger dataset are used. In this work we used the pre-trained models that were trained on the ImageNet dataset which is biggest dataset to our knowledge for such classification.

The system performs a pre-segmentation of the region of interest prior to sending the images to CNN, in an aim to eliminate pathologic features that are present outside of the region spanning from the ONL to Bruch's membrane/inner choroid. Theoretically, it should increase the performance of the system. However, we did not observe any significant improvement that would be relevant in practice. In a post-hoc review of images in our dataset, we found that the B-scans, which had AMD-related pathologic features outside of the ONL to Bruch's membrane/inner choroid region, also had AMD features present within the region of interest. This likely explains why we did not observe any significant improvement.

Since, the number of diseased images in our dataset were significantly less than the number of normal images, to ensure fair learning of the CNNs we considered two different arrangements during training. In the first arrangement all the diseased images were considered, whereas normal images are chosen randomly to match the number of images in the diseased category. Data augmentation was performed for each of the categories. In the second arrangement we performed data augmentation of the diseased images, and randomly chose similar number (after augmentation) of normal images as explained in section the Dataset section. Unsurprising, the accuracy for classification of arrangement -1 (summarized in Table 3) was less than the classification accuracy of arrangement -2 , which meant data augmentation is not fully able to generate all the different scenarios that we observe naturally. That explains why arrangement -2 was considered in implementing the system.

There are limitations to our system. One important limitation ascends by the nature of deep neural networks, in which the network was provided with only the image and associated label, without explicit definitions of features (e.g. SDD, IHRF or hRF). Because the network "learned" the features that were most critical for correct classification, there is a chance that the algorithm is using features previously not recognized or ignored by humans. Another limitation is that the study used images collected from a single clinical site. 


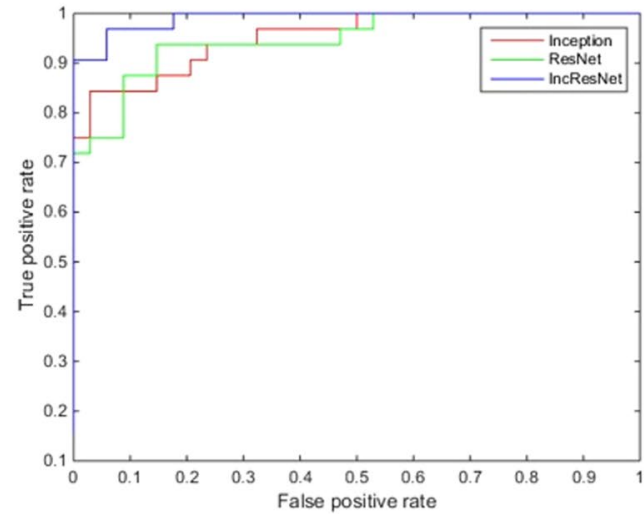

(a)

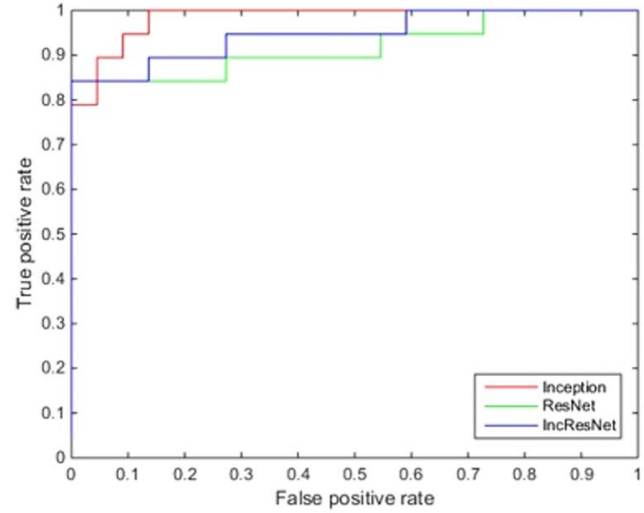

(b)

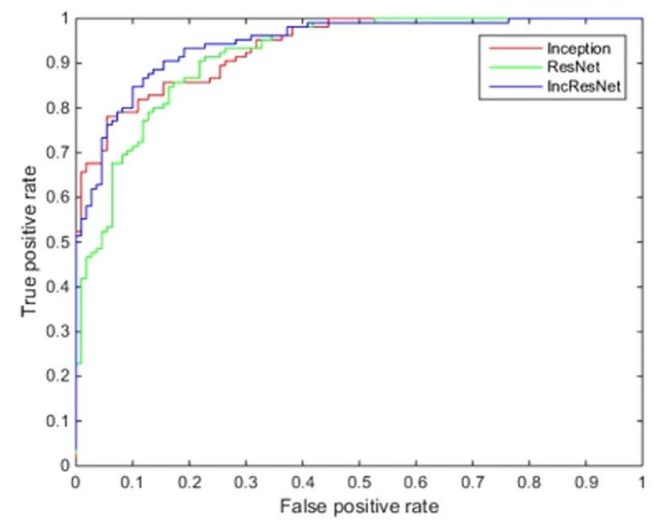

(c)

Figure 5. Receiver operating characteristic (ROC) curve of the three different nets for identifying the presence of (a) IHRF, (b) hRF, and (c) SDD.

\begin{tabular}{|c|c|c|c|c|}
\hline & Sensitivity & Specificity & AUC & Accuracy \\
\hline \multicolumn{5}{|l|}{ IHRF (\%) } \\
\hline Inception-v3 & 81 & 97 & 95 & 89 \\
\hline ResNet50 & 87 & 91 & 95 & 89 \\
\hline InceptionResNet50 & 78 & 100 & 99 & 89 \\
\hline \multicolumn{5}{|l|}{ hRF (\%) } \\
\hline Inception-v3 & 79 & 95 & 98 & 88 \\
\hline ResNet50 & 74 & 100 & 91 & 88 \\
\hline InceptionResNet50 & 84 & 90 & 94 & 88 \\
\hline \multicolumn{5}{|l|}{ SDD (\%) } \\
\hline Inception-v3 & 83 & 85 & 92 & 84 \\
\hline ResNet50 & 96 & 65 & 91 & 80 \\
\hline InceptionResNet50 & 79 & 92 & 94 & 86 \\
\hline
\end{tabular}

Table 2. Sensitivity, specificity, AUC and accuracy obtained by different models.

\begin{tabular}{|l|l|l|l|}
\hline \multirow{2}{*}{ CNNs } & \multicolumn{4}{|l|}{ Accuracy (\%) } \\
\cline { 2 - 4 } & IHRF & hRF & SDD \\
\hline Inception-v3 & 89 & 88 & 84 \\
\hline ResNet50 & 88 & 87 & 81 \\
\hline InceptionResNet50 & 89 & 87 & 85 \\
\hline
\end{tabular}

Table 3. Accuracy obtained by different models. 


\section{Conclusions}

In this study, we sought to develop an effective deep learning method to identify the presence of early AMD biomarkers from OCT images of the retina. We compared the performance of several deep learning networks in an aim to identify the best net in this context. We also incorporated several image pre-processing techniques to improve the classification accuracy. We obtained an accuracy of $86 \%$ to identify the presence of subretinal drusenoid deposit. Intraretinal hyperreflective foci and hyporeflective foci within drusen were detected with an accuracy of $89 \%$ and $88 \%$, respectively. Worth mentioning, the rate of disagreement between different graders are above $20 \%{ }^{9,50}$. An automated system that achieves an accuracy of $86 \sim 89 \%$ with the gold standard, and produces classification with a fraction of time required by an expert grader, is a promising choice to move forward.

We used $90 \%$ of the data for training to ensure the robustness of the algorithm. The results from the $10 \%$ testing data has indicated a good performance. Our clinic is continuously collecting data with new patients and we will further test our algorithm with future new data.

Given the increasing burden of AMD on the healthcare system, the proposed automated system is highly likely to perform a vital role in decision support systems for patient management and in population and primary care-based screening approaches for AMD. With the growing and critical role of OCT in the understanding and monitoring of AMD progression, the proposed automated system should be of clinical value, not only for increasing diagnostic accuracy and efficiency in clinical practice, but also in the design and execution of future early intervention therapeutic trials.

\section{Code Availability}

The code generated during the study is accessible from the corresponding author based on reasonable request and subject to the rule/regulatory of the institute.

\section{References}

1. VanNewKirk, M. R. et al. The Prevalence of Age Related Maculopathy: The Visual Impairment Project. Ophthalmology. 107, 1593-1600 (2000).

2. Bressler, N.M. et al. Potential public health impact of age-related eye disease study results: AREDS report no. 11. Arch Ophthalmol. 121, 1621-1624.

3. Age-Related Eye Disease Study Research Group. A randomized, placebo-controlled, clinical trial of high-dose supplementation with vitamins $\mathrm{C}$ and $\mathrm{E}$, beta carotene, and zinc for age-related macular degeneration and vision loss: AREDS report no. 8. Arch Ophthalmol. 119, 1417-1436 (2001).

4. Jack, L. S. et al. Emixustat and lampalizumab: potential therapeutic options for geographic atrophy. Dev Ophthalmol. 55, 302-309 (2016).

5. Mitchell, P. et al. Prevalence of agerelated maculopathy in Australia. The Blue Mountains Eye Study. Ophthalmology. 102, 1450-1460 (1995).

6. Klein, R. et al. The five-year incidence and progression of age-related maculopathy: the Beaver Dam Eye Study. Ophthalmology. 104(6), 7-21 (1997).

7. Davis, M. D. et al. The age-related eye disease study severity scale for age-related macular degeneration: AREDS report no. 17. Arch Ophthalmol. 123, 1484-1498 (2005).

8. Ferris, F. L. et al. A simplified severity scale for age-related macular degeneration: AREDS report no. 18. Arch Ophthalmol. 123, 1570-1574 (2005).

9. Lei, J. et al. Proposal of a simple optical coherence tomography-based scoring system for progression of age-related macular degeneration. Graefe's Archive for Clinical and Experimental Ophthalmology. 255(8), 1551-8 (2017).

10. Abdelfattah, N. S. et al. Drusen volume as a predictor of disease progression in patients with late age-related macular degeneration in the fellow eye. Invest Ophthalmol Vis Sci. 57, 1839-1846 (2016).

11. Nassisi, M. et al. Quantity of Intraretinal Hyperreflective Foci in Patients With Intermediate Age-Related Macular Degeneration Correlates With 1-Year Progression. Investigative ophthalmology \& visual science. 59(8), 3431-9 (2018).

12. Ouyang, Y. et al. Optical coherence tomography-based observation of the natural history of drusenoid lesion in eyes with dry agerelated macular degeneration. Ophthalmology. 120, 2656-2665 (2013).

13. Finger, R. P. et al. Reticular pseudodrusen: a risk factor for geographic atrophy in fellow eyes of individuals with unilateral choroidal neovascularization. Ophthalmology. 121, 1252-1256 (2014).

14. Marsiglia, M. et al. Association between geographic atrophy progression and reticular pseudodrusen in eyes with dry age-related macular degeneration. Invest Ophthalmol Vis Sci. 54, 7362-7369 (2013).

15. Zhou, Q. et al. Pseudodrusen and incidence of late age-related macular degeneration in fellow eyes in the comparison of age-related macular degeneration treatments trials. Ophthalmology. 123, 1530-1540 (2016).

16. Jain, N. et al. Quantitative comparison of drusen segmented on SD-OCT versus drusen delineated on color fundus photographs. Investigative ophthalmology \& visual science. 51(10), 4875-83 (2010).

17. Farsiu, S. et al. Fast detection and segmentation of drusen in retinal optical coherence tomography images. Ophthalmic Technologies XVIII (Vol. 6844, p. 68440D). International Society for Optics and Photonics (Feb 11 2008).

18. Chen, Q. et al. Automated drusen segmentation and quantification in SD-OCT images. Medical image analysis. 17(8), 1058-72 (2013).

19. de Sisternes, L. et al. Quantitative SD-OCT imaging biomarkers as indicators of age-related macular degeneration progression. Investigative ophthalmology \& visual science. 55(11), 7093-103 (2014).

20. Iwama, D. et al. Automated assessment of drusen using three-dimensional spectral-domain optical coherence tomography. Investigative ophthalmology \& visual science. 53(3), 1576-83 (2012).

21. Diniz, B. et al. Drusen measurements comparison by fundus photograph manual delineation versus optical coherence tomography retinal pigment epithelial segmentation automated analysis. Retina. 34(1), 55-62 (2014).

22. Gregori, G. et al. Spectral domain optical coherence tomography imaging of drusen in nonexudative age-related macular degeneration. Ophthalmology. 118(7), 1373-9 (2011).

23. Chen, Q. et al. Semi-automatic geographic atrophy segmentation for SD-OCT images. Biomed Opt Express. 4, 2729-2750 (2013).

24. Yehoshua, Z. et al. Comparison of geographic atrophy measurements from the oct fundus image and the sub-RPE slab image. Ophthalmic Surg Lasers Imaging Retina. 44, 127-132 (2013).

25. Tsechpenakis, G. et al. Geometric deformable model driven by CoCRFs: application to optical coherence tomography. Med Image Comput Comput Assist Interv. 11, 883-891 (2008).

26. $\mathrm{Hu}, \mathrm{Z}$. H. et al. Segmentation of the geographic atrophy in spectral-domain optical coherence tomography and fundus autofluorescence images. Invest Ophthalmol Vis Sci. 54, 8375-8383 (2013). 
27. Ahlers, C. et al. Automatic segmentation in three-dimensional analysis of fibrovascular pigmentepithelial detachment using highdefinition optical coherence tomography. Br J Ophthalmol. 92, 197-203 (2008).

28. Penha, F. M. et al. Quantitative imaging of retinal pigment epithelial detachments using spectral-domain optical coherence tomography. Am J Ophthalmol. 153, 515-523 (2012).

29. Sun, Z. et al. An automated framework for 3D serous pigment epithelium detachment segmentation in SD-OCT images. Sci Reports. 6, $21739(2016)$

30. Shi, F. et al. Automated 3-D retinal layer segmentation of macular optical coherence tomography images with serous pigment epithelial detachments. IEEE Trans Med Imaging. 34, 441-452 (2014).

31. Pilch, M. et al. Automated segmentation of pathological cavities in optical coherence tomography scans. Invest Ophthalmol Vis Sci. 54, 4385-4393 (2013).

32. Zheng, Y. et al. Computerized assessment of intraretinal and subretinal fluid regions in spectral-domain optical coherence tomography images of the retina. Am J Ophthalmol. 155, 277-286 (2013).

33. Fernandez, D. C. Delineating fluid-filled region boundaries in optical coherence tomography images of the retina. IEEE Trans Med Imag. 24, 929-945 (2005).

34. Chiu, S. J. et al. Validated automatic segmentation of AMD pathology including drusen and geographic atrophy in SD-OCT images. Invest Ophthalmol Vis Sci. 53, 53-61 (2012).

35. Wintergerst, M. W. M. et al. Algorithms for the Automated Analysis of Age-Related Macular Degeneration Biomarkers on Optical Coherence Tomography: A Systematic Review. Translation Vision Science and Technology. 6(4), 1-20 (2017).

36. Schmidt-Erfurth, U. et al. Prediction of Individual Disease Conversion in Early AMD Using Artificial Intelligence. Investigative ophthalmology \& visual science. 59(8), 3199-208 (2018).

37. Lee, S. Y. et al. Automated characterization of pigment epithelial detachment by optical coherence tomography. Invest Ophthalmol Vis Sci. 53, 164-170 (2012).

38. Ho, J. J. et al. Documentation of intraretinal retinal pigment epithelium migration via high-speed ultrahigh-resolution optical coherence tomography. Ophthalmology. 118, 687-693 (2011).

39. LeCun, Y. et al. Deep learning. Nature [Internet]. 521(7553), 436-444, https://doi.org/10.1038/nature14539\%5Cn10.1038/ nature14539 (2015)

40. Gulshan, V. et al. Development and validation of a deep learning algorithm for detection of diabetic retinopathy in retinal fundus photographs. JAMA. 316(22), 2402-10 (2016).

41. Saha, S. K et al. Automated Quality Assessment of Colour Fundus Images for Diabetic Retinopathy Screening in Telemedicine. Journal of digital imaging. (Apr 27), 1-10, https://doi.org/10.1007/s10278-018-0084-9 (2018).

42. Diaz-Pinto, A. et al. CNNs for automatic glaucoma assessment using fundus images: an extensive validation. Biomedical engineering online. 18(1), 29 (2019).

43. Tajbakhsh, N. et al. Convolutional neural networks for medical image analysis: full training or fine tuning? IEEE transactions on medical imaging. 35(5), 1299-1312 (2016).

44. Deng, J. et al. ImageNet: a large-scale hierarchical image database. IEEE Computer Vision and Pattern Recognition. 248-255 (2009).

45. Szegedy, C. et al. Rethinking the inception architecture for computer vision. Proceedings of the IEEE conference on computer vision and pattern recognition. $2818-2826$ (2016).

46. He, K. et al. Deep residual learning for image recognition. Proceedings of the IEEE conference on computer vision and pattern recognition. 770-778 (2016).

47. Szegedy, C. et al. Inception-v4, inception-resnet and the impact of residual connections on learning. AAAI. 4, 12 (2017).

48. Roy, A. G. et al. ReLayNet: retinal layer and fluid segmentation of macular optical coherence tomography using fully convolutional networks. Biomedical optics express. 8(8), 3627-42 (2017)

49. Saha, S. K. et al. Deep learning for automatic detection and classification of microaneurysms, hard and soft exudates, and hemorrhages for diabetic retinopathy diagnosis. Investigative Ophthalmology \& Visual Science. 57(12), 5962 (2016).

50. Christopher, M. et al. Performance of Deep Learning Architectures and Transfer Learning for Detecting Glaucomatous Optic Neuropathy in Fundus Photographs. Scientific reports. 8(1), 16685 (2018).

51. Kermany, D. S. et al. Identifying medical diagnoses and treatable diseases by image-based deep learning. Cell. 172(5), 1122-31 (2018).

\section{Acknowledgements}

This work was supported, in part, by NIH-NEI grant R21-EY030619, an award from Australia Endeavour Scholarships and Fellowships, a grant from the BrightFocus Foundation Macular Degeneration program, and a grant from Macula Vision Research Foundation (MVRF).

\section{Author Contributions}

Sajib Saha developed the system, performed experiments, and wrote the main manuscript text. Marco Nassisi, Mo Wang, and Sophiana Lindenberg performed the manual assessment of the biomarkers for ground truth. Yogi Kanagasingam and Srinivas Sadda wrote part of the manuscript. Zhihong Jewel Hu cultivated the idea of the development, and also wrote part of the manuscript.

\section{Additional Information}

Competing Interests: The authors declare no competing interests.

Publisher's note: Springer Nature remains neutral with regard to jurisdictional claims in published maps and institutional affiliations.

Open Access This article is licensed under a Creative Commons Attribution 4.0 International License, which permits use, sharing, adaptation, distribution and reproduction in any medium or format, as long as you give appropriate credit to the original author(s) and the source, provide a link to the Creative Commons license, and indicate if changes were made. The images or other third party material in this article are included in the article's Creative Commons license, unless indicated otherwise in a credit line to the material. If material is not included in the article's Creative Commons license and your intended use is not permitted by statutory regulation or exceeds the permitted use, you will need to obtain permission directly from the copyright holder. To view a copy of this license, visit http://creativecommons.org/licenses/by/4.0/.

(C) The Author(s) 2019 